\title{
Physics aspects of prostate tomotherapy: Planning optimization and image-guidance issues
}

\author{
Claudio Fiorino, Filippo Alongi, Sara Broggi, Giovanni Mauro Cattaneo, \\ Cesare Cozzarini, Nadia Di Muzio, Eleonora Maggiulli, Paola Mangili, Lucia \\ Perna, Riccardo Valdagni, Ferruccio Fazio \& Riccardo Calandrino
}

To cite this article: Claudio Fiorino, Filippo Alongi, Sara Broggi, Giovanni Mauro Cattaneo, Cesare Cozzarini, Nadia Di Muzio, Eleonora Maggiulli, Paola Mangili, Lucia Perna, Riccardo Valdagni, Ferruccio Fazio \& Riccardo Calandrino (2008) Physics aspects of prostate tomotherapy: Planning optimization and image-guidance issues, Acta Oncologica, 47:7, 1309-1316, DOI: $10.1080 / 02841860802266755$

To link to this article: https://doi.org/10.1080/02841860802266755

曲 Published online: 08 Jul 2009.

Submit your article to this journal $\pi$

Џlll Article views: 938

Q View related articles $\sqsubset$

4 Citing articles: 3 View citing articles 준 


\title{
Physics aspects of prostate tomotherapy: Planning optimization and image-guidance issues
}

\author{
CLAUDIO FIORINO ${ }^{1}$, FILIPPO ALONGI ${ }^{2}$, SARA BROGGI ${ }^{1}$, GIOVANNI MAURO \\ CATTANEO $^{1}$, CESARE COZZARINI ${ }^{2}$, NADIA DI MUZIO ${ }^{2}$, ELEONORA MAGGIULLI ${ }^{1}$, \\ PAOLA MANGILI ${ }^{1}$, LUCIA PERNA ${ }^{1}$, RICCARDO VALDAGNI ${ }^{3}$, FERRUCCIO FAZIO $^{2}$ \& \\ RICCARDO CALANDRINO ${ }^{1}$
}

${ }^{1}$ Medical Physics, S. Raffaele Institute, Milano, Italy, ${ }^{2}$ Department of Radiotherapy, S. Raffaele Institute, Milano, Italy and

${ }^{3}$ Prostate Program, National Institute of Cancer, Milano, Italy

\begin{abstract}
Purpose. To review planning and image-guidance aspects of more than 3 years experience in the treatment of prostate cancer with Helical Tomotherapy (HT). Methods and materials. Planning issues concerning two Phase I-II clinical studies were addressed: in the first one, 58 Gy in 20 fractions were delivered to the prostatic bed for post-prostatectomy patients: in the second one, a simultaneous integrated boost (SIB) approach was applied for radical treatment, delivering 71.4-74.2 Gy to the prostate in 28 fractions. On-line daily MVCT image guidance was applied: bone match was used for post-operative patients while prostate match was applied for radically treated patients. MVCT data of a large sample of both categories of patients were reviewed. Results. At now, more than 250 patients were treated. Planning data show the ability of HT in creating highly homogeneous dose distributions within PTVs. Organs at risk (OAR) sparing also showed to be excellent. HT was also found to favorably compare to inversely-optimized IMAT in terms of PTVs coverage and dose distribution homogeneity. In the case of pelvic nodes irradiation, a large sparing of bowel was evident compared to 3DCRT and conventional 5-fields IMRT. The analysis of MVCT data showed a limited motion of the prostate (about $5 \%$ of the fractions show a deviation $\geq 3 \mathrm{~mm}$ in posterior-anterior direction), due to the careful application of rectal emptying procedures. Based on phantom measurements and on the comparison with intra-prostatic calcification-based match, direct visualization prostate match seems to be sufficiently reliable in assessing shifts $\geq 3 \mathrm{~mm}$. Conclusions. HT offers excellent planning solutions for prostate cancer, showing to be highly efficient in a SIB scenario. Daily MVCT information showed evidence of a limited motion of the prostate in the context of rectal filling control obtained by instructing patients in selfadministrating a rectal enema.
\end{abstract}

The recent advent of Image-guided Radiotherapy (IGRT) for the treatment of prostate cancer is mainly due to the proximity of the prostate to organs at risk, primarily the rectum, in a "moving" organ scenario. Helical Tomotherapy (HT) [1] is an innovative intensity-modulated IGRT technique that can find interesting applications in the treatment of prostate carcinoma; the helical delivery permits to obtain highly tailored dose distributions with excellent coverage and homogeneity within different targets, especially in the case of concomitant boost delivery.
The highly efficient integration of image guidance through Megavoltage computed tomography (MVCT) suggests the feasibility of implementing on-line correction strategies for both radical and post-operative patients.

HT was installed at our Institute in 2004 and moderate hypofractionated clinical protocols for prostate Tomotherapy were activated in 2005 . Up to now more than 250 patients were treated and first clinical results concerning acute and early late toxicity have been published $[2,3]$.

Correspondence: Claudio Fiorino, Medical Physics, H. S. Raffaele, Via Olgettina 60, 20132 Milano, Italy. Tel: +39 226432278. Fax: +39 226432773. E-mail: fiorino.claudio@hsr.it 
In this paper, we review our experience in planning and image-guidance, focusing on the patients treated within two phase I-II clinical studies.

\section{Materials and methods}

Phase I-II studies in progress

Two different phase I-II clinical studies exploring hypofractionation were activated in the last years, one for adjuvant (TOMOADV) and one for radical (TOMOSIB) treatment.

In the TOMOADV trial, the clinical target volume (CTV) included the tumour bed; clinical data and location of surgical clips were also used to guide the physician in contouring the CTV that included the prostatic fossa and the lower bladder neck; inferiorly, the CTV was drawn to about $1-1.5 \mathrm{~cm}$ from the caudal limit of the ischiatic tuberosities. Bony structures and the anterior rectal wall were also used to define the edges of CTV.

For pT3b patients 2 different CTVs were drawn as follows: CTV1 was the prostatic plus seminal vesicle bed, while CTV2 included the prostate bed only, being CTV1 usually extended $1.5-2.5 \mathrm{~cm}$ above the cranial limit of CTV2. Clinical target volume and PTV definitions were consistent with the irradiated volumes in our conventionally fractionated 3DCRT experience: 8,10 and $8 \mathrm{~mm}$ margins were used for left-right, cranial-caudal and anterior-posterior direction respectively.

Fifty-eight Gy in 20 fractions (2.9 Gy/fr) were prescribed to PTV2 (the prostatic bed), whereas for pT3b patients, $53 \mathrm{~Gy}(2.65 \mathrm{~Gy} / \mathrm{fr})$ were delivered to PTV1, concomitantly delivering 58 Gy to CTV2.

This fractionation scheme was chosen in order to deliver a treatment which must be at the same time safe (in terms of both acute and late toxicity) and effective enough in terms of $2 \mathrm{~Gy}$ equivalent dose (EQD2, [4]), for $\alpha / \beta$ ratio between 1.5 and $10 \mathrm{~Gy}$. In fact, EQD2 ranges from $62.4 \mathrm{~Gy}(\alpha / \beta=10 \mathrm{~Gy})$ to 73 Gy $(\alpha / \beta=1.5 \mathrm{~Gy})$, i.e. within the range of doses conventionally delivered in high-dose post-operative radiotherapy, with the hope of a moderate escalation of EQD2 compared to the EQD2 normally delivered with conventional $70.2 \mathrm{~Gy} / 1.8 \mathrm{~Gy} / \mathrm{fr}$ schedule, if $\alpha / \beta=1.5$ Gy. On the other hand, the EQD2 for rectum and bladder $(\alpha / \beta=3 \mathrm{~Gy}$, late toxicity) resulted to be below $70 \mathrm{~Gy}$.

In the TOMOSIB study, according to National Comprehensive Cancer Network (NCCN) we identified three risk groups: Low risk (clinical stage T1$\mathrm{T} 2$, Gleason score $\leq 6$, PSA $\leq 10$ ); Intermediate risk (clinical stage T1-T2, Gleason score $\leq 6$, PSA $>10$ or clinical stage T1-T2, Gleason $>6$, PSA $\leq 10$, or clinical stage T3, Gleason score $<6$, PSA $<10$ ); High risk (clinical stage T1-T3, Gleason score $\geq 7$, PSA $>$ 10). Five different target structures were defined: the pelvic nodes (CTV1), including obturator, hypogastric, external and internal iliac and pre-sacral lymphnodes, anterior to the first sacral segment, deriving the position of the nodes from published atlas; the most cranial portion of the seminal vesicles (CTV2); the first third of the seminal vesicles (CTV3); the prostate (CTV4); the overlap between the highest dose PTV and the rectum (OVERLAP).

Planning target volumes were defined as follows: concerning CTV2-4, 8, 10 and $8 \mathrm{~mm}$ margins were used for left-right, cranial-caudal and anteriorposterior direction respectively; for pelvic lymph nodes $7-10 \mathrm{~mm}$ in all directions excepting the portion close to the bony structures where the margins were decreased to $5 \mathrm{~mm}$. The margins applied are similar to the ones applied in 3DCRT, with the only exception of lymph nodes, where a wider margin was used for HT, because of potential miss due to the use of MVCT guide in order to track the movements of the prostate.

The scheduled doses relative to the various targetstructures in the different classes of risk are reported in Table I. Considering that the true value of $\alpha / \beta$ ratio is unknown, our fractionation scheme was chosen in order to deliver a $2 \mathrm{~Gy}$ equivalent dose (EQD2) at least not inferior to the ones routinely delivered at our Institution with conventional fractionation (74-78 Gy). In Table II the EQD2 delivered with our protocol are shown for $\alpha / \beta$ ratio ranging from 1.5

Table I. Prescribed doses (in Gy) for different PTVs in our simultaneous integrated boost approach of the moderately hypofractionated trial, named TOMOSIB. According with NCCN risk class assessment different doses are delivered to the PTVs referred to pelvic nodes (only for intermediate/high risk patients), upper and lower seminal vesicles, prostate and overlap between highest-dose PTVs and rectum.

\begin{tabular}{|c|c|c|c|c|c|c|}
\hline \multirow[b]{2}{*}{ Volumes of interest } & \multicolumn{2}{|c|}{ Low risk NCCN } & \multicolumn{2}{|c|}{ Intermediate risk NCCN } & \multicolumn{2}{|c|}{ High risk NCCN } \\
\hline & Dose/fr. & Total dose & Dose/fr. & Total dose & Dose/fr. & Total dose \\
\hline PTV1 (nodes) & & & 1.85 & 51.8 & 1.85 & 51.8 \\
\hline PTV2 (upper SV) & 2 & 56 & 2.2 & 61.6 & 2.34 & 65.5 \\
\hline PTV3 (lower SV) & 2.2 & 61.6 & 2.34 & 65.5 & 2.65 & 74.2 \\
\hline PTV4 (prostate) & 2.55 & 71.4 & 2.65 & 74.2 & 2.65 & 74.2 \\
\hline OVERLAP & 2.34 & 65.5 & 2.34 & 65.5 & 2.34 & 65.5 \\
\hline
\end{tabular}


Table II. The 2-Gy Equivalent doses (EQD2) referred to the TOMOSIB prescribed dose reported in Table I, are shown. TOMOSIB is planned to deliver EQD2 ranging in the routinely delivered dose for high $\alpha / \beta$ values with the hope of substantial dose escalation in the case $\alpha / \beta$ is low. At the same time, the EQD2 for the portion of rectum overlapped with PTVs is kept below 70 Gy ( $\alpha / \beta=3$, late toxicity).

\begin{tabular}{|c|c|c|c|c|c|c|}
\hline Volumes of interest & Dose/fr. & nominal dose & $\mathrm{EQD} 2 \alpha / \beta=1.5$ & $\mathrm{EQD} 2 \alpha / \beta=3$ & $\mathrm{EQD} 2 \alpha / \beta=10$ & $\mathrm{EQD} 2 \alpha / \beta=15.5$ \\
\hline Pelvic nodes & 1.85 & 51.8 & 49.6 & 50.2 & 51.1 & 51.4 \\
\hline Upper SV & 2 & 56 & 56 & 56 & 56 & 56 \\
\hline Lower SV & 2.2 & 61.6 & 65.1 & 64.1 & 62.6 & 62.3 \\
\hline Upper/lower SV OVERLAP & 2.34 & 65.5 & 71.9 & 70 & 67.4 & 66.8 \\
\hline lower SV prostate & 2.55 & 71.4 & 82.6 & 79.3 & 74.7 & 73.6 \\
\hline prostate & 2.65 & 74.2 & 88 & 83.8 & 78.2 & 77 \\
\hline
\end{tabular}

and 15.5 Gy: the dose to the overlap has been set to $65.5 \mathrm{~Gy}$, corresponding to an $\mathrm{EQD} 2(\alpha / \beta=3 \mathrm{~Gy})$ equal to 70 Gy that can be considered as safe from the point of view of late rectal toxicity $[5,6]$. The value of 15.5 was recently suggested as the most likely value for hypoxic tumors [7].

\section{Adjuvant hypofractionated protocol: Planning issues}

Inverse optimization with HT is very different from the optimization of conventional IMRT delivered with a linear accelerator.

Three parameters can be set by the operator: field width, pitch and modulation factor. Briefly, the field width is the fixed field dimension in the cranialcaudal direction (to be chosen between 1, 2.5 and $5 \mathrm{~cm}$ ); the pitch is the ratio between the couch translation during one gantry rotation and the field width; the modulation factor is the ratio between maximum and average beam intensity.

Small field dimensions, small pitch and large modulation factors mean longer time of irradiation and greater ability of the delivery system to sculpt complex dose distribution with steeper dose gradients [8].

For most patients a field dimension of $2.5 \mathrm{~cm}$, a pitch of 0.287 and a modulation factor of 2-2.5 were used: the resulting irradiation times were typically in the range of 4-5 minutes.

For all patients, the dose was prescribed in terms of median dose to PTV.

Concerning PTV, the goal was to deliver more than $55.1 \mathrm{~Gy}$ ( $95 \%$ of the prescribed dose) to more than $95 \%$ of the volume, while keeping dose homogeneity as high as possible. Dmax in the PTV was set to be below $105 \%$ of the prescribed dose.

Concerning rectum and bladder, no attempts were made to reduce the dose in the overlap region between these organs and the PTV; in the regions outside the overlap, the planner attempted to reduce the dose "as much as possible", starting from the fraction of rectum/bladder receiving "high" dose and passing to intermediate and low doses.
Concerning rectum sparing, following the previously described optimisation method, the resulting plan was expected to largely satisfy the dose constraints normally used for 3DCRT. These values were derived from previous investigations $[5,6]$ and scaled to approximately take into account the non conventional dose fractionation through the linear quadratic model ("safe" estimate gives: V $55<30$; $\mathrm{V} 50<40 ; \mathrm{V} 40<60$, where $\mathrm{VX}$ is the fraction of organ receiving at least $\mathrm{X} G \mathrm{G}$ ).

Femoral heads (and femurs) were set to receive a maximum dose below 35 Gy while reducing the fraction of organ receiving more than $20 \mathrm{~Gy}$ to a small percentage. No hot spot ( $>50 \mathrm{~Gy}$ ) outside the PTV, excluding the first surrounding $1-2 \mathrm{~cm}$, was admitted.

DVH-dose statistical data for the first 50 patients were collected: mean values, SD and range of several dosimetry parameters were calculated [3].

\section{Simultaneous integrated boost for radically treated patients: Planning issues}

For most patients a field dimension of $2.5 \mathrm{~cm}$, a pitch of 0.287 and a modulation factor of 3-3.5 were used. The resulting irradiation times typically were in the range of 4 to 8 minutes. For all patients, the dose was prescribed as median dose to PTV4 while V68/70 $>95 \%$ and Dmax $<74 / 77$ for low and intermediate/high risk respectively. Concerning PTV1-3, the goal was to deliver more than $98 \%$ of the prescribed dose to more than $95 \%$ of the volume while keeping dose homogeneity as high as possible. The median dose in the overlap was set to be 65.5 Gy while satisfying V64 $>95 \%$ and Dmax $<70$ Gy. Concerning the rectum outside the overlap, the planner forced the system to reduce the dose "as much as possible", starting from the fraction of the rectum receiving "high" dose and then passing to intermediate and low doses, similarly to the TOMOADV patients.

Concerning the bladder, a similar approach was followed but without any attempt to reduce the dose in the overlap between PTV1-4 and bladder. 
Femoral heads (and femurs) were set to receive a maximum dose below $50 \mathrm{~Gy}$ while reducing the fraction of organ receiving more than 35-40 Gy to a very low percentage.

No hot spot ( $>50$ Gy) outside the PTV, excluding the first $1-2 \mathrm{~cm}$ surrounding it, was admitted. In the case that the bulb was drawn, the planner tried to reduce the dose to as great an extent as possible without reducing the coverage of the PTV on the most caudal slice (i.e.: the $95 \%$ isodose should encompass the PTV on this slice).

Concerning the intestinal cavity (IC), the planner was asked to reduce the dose without compromising PTV coverage, starting from the fraction of IC receiving more than 40-50 Gy, and subsequently passing gradually to the fraction of IC receiving more than 20-30 Gy. Planning data of the first 30 patients were recently reviewed [9].

\section{Pelvic nodes tomotherapy}

Up to now, more than 70 patients were irradiated with HT for prostate cancer including pelvic nodes. Part of them were enrolled in the TOMOSIB $(\mathrm{n}=36)$ while the remaining were irradiated for adjuvant/salvage intent while concomitantly delivering 65.5-72 Gy in 25-30 fractions to the prostatic bed. Due to the peculiar ability of HT in dramatically sparing the intestinal cavity, planning data referred to pelvis irradiation were recently reviewed [2], independently on the treatment intent. These data were compared with a cohort of patients treated in the same period (2004-2007) with conventional box-technique and with 5-fields IMRT with dynamic MLC (Varian system, EclipseHelios + DHX Linac); dose-volume information was also correlated with acute upper GI toxicity. The intestinal cavity (IC) was defined according with the definition recently reported by Sanguineti et al. [10], excepting for the fact that we exclude the fraction of IC overlapped to PTV, keeping a $5 \mathrm{~mm}$ margin between IC and PTV.

Image guidance for post-operative patients: Bone match on- and off-line correction strategies

Concerning image-guidance with MVCT, all TOMOADV patients were daily imaged in the normal mode, that means that during one gantry rotation the couch translates for $2 \mathrm{~mm}$; two slices are reconstructed in one rotation. After the initial patient setup based on tattoo marks, a MVCT scan was acquired along the total PTV length (10-15 slices). For patient alignment current MVCT images were automatically registered with the planning kVCT images by using the bone matching automatic registration technique. Only translational corrections were applied to the patient position. Organ motion was deliberately ignored as the impact of rectal filling on the position of the prostatic bed has not yet been clarified. For this reason we routinely apply the same margins used for radically treated patients. Due to the lower dose delivered compared to radically treated patients and to the uncertainty in defining the CTV for post-prostatectomy patients margin reduction was not considered as a major aim of image-guide, being potentially dangerous too. We limited the use of MVCT just to reduce as much as possible any risk of geographical miss for these patients. MVCT data referred to 37 patients were recently reviewed [11].

Image guidance for radically treated patients: Prostate match on-line correction strategy

As for post-prostatectomy patients, daily MVCT image-guidance was applied for all TOMOSIB patients; the normal mode is routinely used.

A two-step matching strategy was followed: first, a fully automatic registration based on bony anatomy (Bone matching, BM) was carried out: the uncertainty of the automatic bone matching was investigated on appropriate phantoms [11] and was found to be highly efficient and accurate $(<1 \mathrm{~mm})$.

After BM, the matching was adjusted by a physician through direct visualization (DV) of the prostate, quite consistently with a prostate center-ofmass shift; based on the direct visualization of prostate anatomy, the physician matches the actual position of the prostate with the planning $\mathrm{kVCT}$ one, without any contouring procedure. After the accomplishment of the matching procedures, the final correction was automatically applied for translations and roll angle rotation (axis of rotation: head-feet) and the patient was subsequently treated.

For each fraction, the values of the shifts after BM (set-up error) and after BM +DV (total error: setup + organ motion) were registered for the three main directions (left-right: LR; posterior-anterior: PA; cranial-caudal: CC) and for the roll angle. For all patients, rectum emptying through daily enema before planning scan and each morning during therapy was prescribed in order to limit prostate motion and possible organ deformation.

Data referred to 21 low-risk patients were recently reviewed [12]. In order to assess the intrinsic uncertainty of our two-step matching strategy $(\mathrm{BM}+\mathrm{DV})$, we also compared the prostate shift assessed with $\mathrm{BM}+\mathrm{DV}$ with the match based on intra-prostatic calcifications (used as "natural markers") on 20 randomly chosen MVCTs of 5 patients. 


\section{Results}

TOMOADV patients: Planning data

Planning data referred to the first 50 patients have recently been reviewed [2]. In summary, a very low mean rectal dose was obtained (median value: 26.5 Gy) with V55 ranging between 5 and $14 \%$. Bladder Dmean was 39.7 Gy (median) while femoral heads were highly spared (V20 ranged between 0 and $11 \%)$. Highly homogeneous dose distributions within PTV were obtained with V55 ranging between 96 and $99 \%$ and Dmax between 59.5 and 63 Gy (median: $61 \mathrm{~Gy}$ ).

\section{TOMOSIB patients: Planning data}

Planning data referred to the first 60 patients were also recently reviewed [9]. Highly homogeneous dose distributions within the different PTVs were obtained while excellent sparing for the OARs was reached. In particular, V65 of the rectum was $10.2 \%$ (median value) and V60 of the bladder was $14 \%$ (when excluding patients with an empty bladder at the planning CT). Dmean of the penile bulb was 30.5 Gy (median value) while a large sparing of the intestinal cavity was obtained and will be discussed in the next section. Excellent PTV coverage and homogeneity of the dose distribution within PTVs were found: average values of V68/V70 for PTV4 respectively for low and high/intermediate risk patients were respectively 96 and $95 \%$, ranging between 93 and $98 \%$.

\section{Pelvic nodes treatment: Intestinal cavity sparing}

In order to better assess the impact of HT on IC sparing, planning data of 191 patients receiving pelvic nodes irradiation and treated in the same period (2004-2007) with conventional 4-fields $(\mathrm{n}=91)$ as well as Linac IMRT $(n=26)$ and HT $(n=58)$ were recovered. In Figure 1 the "absolute" (i.e.: in cc) average DVHs of the IC (outside PTV) for the three modalities are plotted. The differences between IMRT and 3D and between HT and 3D are highly significant $(\mathrm{p}<0.001$, Mann-Withney U-test) for V25-V50, while V10-V15 are significantly lower $(\mathrm{p}<0.02)$ for $3 \mathrm{D}$ patients. Moreover, HT was significantly better than IMRT in the range V20V35 $(\mathrm{p}<0.001)$.

\section{Image-guidance: Adjuvant patients (bone match)}

HT was found to be very efficient in the use of daily image guidance and set-up correction. Results referred to 28 patients with on-line correction (without action level) showed that the residual error (i.e.: the expected systematic error for the remaining part of

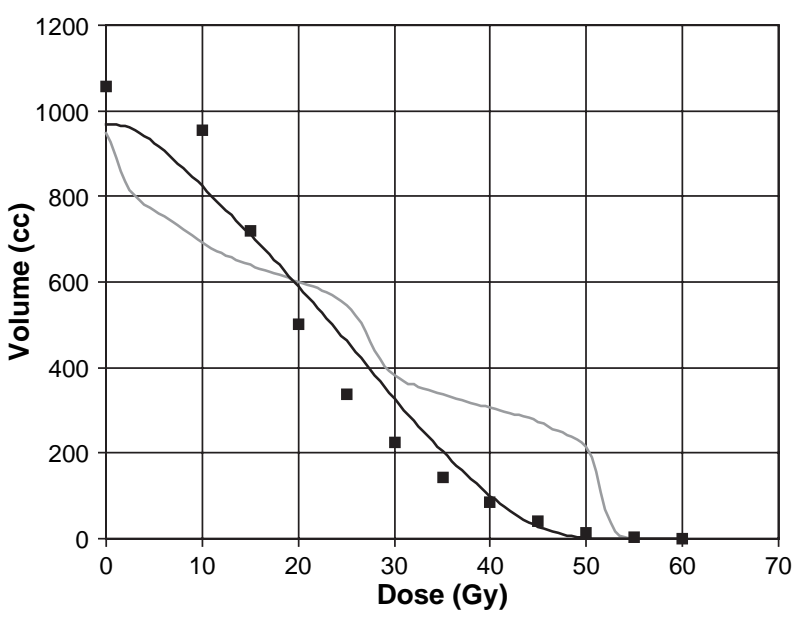

Figure 1. The average DVHs of the intestinal cavity (outside PTV) of patients treated with whole pelvis RT are plotted according to the technique of treatment: conventional 3DCRT box-technique (gray, $\mathrm{n}=91$ ); Linac IMRT with dynamic MLC (black continuous, $\mathrm{n}=26$ ); Helical Tomotherapy (squares, $\mathrm{n}=48$ ).

the treatment when assessing systematic set-up error based on the data of the first $\mathrm{n}$ fractions) reached its minimum at the $7^{\text {th }}-10^{\text {th }}$ fraction, showing that offline correction assessed after the first 3-4 fraction may be slightly detrimental. For instance, the residual systematic error for the posterior-anterior direction resulted to be 2.0 and $1.0 \mathrm{~mm}(1 \mathrm{SD})$ if correcting the systematic error at the first or at the $8^{\text {th }}$ fraction respectively. Anyway, off-line correction after 4 fractions was demonstrated to be able in reducing systematic error below $1.5 \mathrm{~mm}$ (1SD, [11]).

Image-guidance: Organ motion assessed by daily MVCT

The analysis of MVCT data of 21 low-risk TOMOSIB patients showed evidence of very limited motion of the prostate [12] relative to bony anatomy. This surprising result was mainly due to the practice of instructing patients in emptying the rectum by daily rectal enema and, secondarily, prescribing a "full" bladder.

Daily MVCT was found to be suitable in detecting those fractions where a full rectum significantly shifted and/or deformed the prostate (around 5\% of all considered fractions); in this case the patient was treated just after applying actions to "normalize" the rectum (i.e.: patient to the toilet, air emptying after catheter stimulation). These procedures were found to further reduce the impact of prostate motion: the systematic and random errors for organ motion (relative to bony anatomy) were $0.44 / 1.3 \mathrm{~mm}$ and $0.34 / 0.9 \mathrm{~mm}$ respectively without and with the application of post-MVCT rectal emptying procedures [12]. 
The intrinsic uncertainty of the system in assessing prostate position was assessed with a number of experiments on phantoms to estimate the BM accuracy that was found within $0.6 \mathrm{~mm}$ (1 SD). The accuracy of DV compared to intra-prostatic calcifications based match (IPC) showed that DV is not suitable in detecting shift $<3 \mathrm{~mm}$ : on $20 \mathrm{MVCT}$ scan of 5 patients, an average difference equal to 1.2 $\mathrm{mm}$ between DV and IPC match was found with DV underestimating small shift. This was due to the fact that after automatic BM, the operator is not confident in assessing small shift $(<3 \mathrm{~mm})$ through DV.

\section{Discussion and conclusions}

Helical Tomotherapy is an integrated image-guided IMRT system which finds interesting applications in the treatment of prostate cancer $[2,3,9,13-15]$.

Thanks to the physical properties of helical fanbeam delivery it was found to be highly efficient in the concomitant delivery of different levels of dose to different target volumes within a moderate hypofractionated approach.

As reported in some published papers $[13,15,16]$ HT compares favourably with respect to conventional IMRT and also to inverse-planned IMAT when considering the DVHs within PTVs as HT is able to generate very homogeneous dose distributions within each target volume, creating very deep gradient between different PTVs receiving different doses and between PTVs and OARs. In particular, our TOMOSIB approach enhances these potentialities, showing the ability of HT in differentiating between small PTVs (for instance overlap vs prostate).

As reported by Iori et al. [13], HT was found to favourably compare with respect to inverselyoptimized intensity-modulated arc therapy (IMAT) in a recent investigation on 6 low-risk patients (without pelvis irradiation) when considering PTVs coverage and homogeneity of the dose distribution while assuring similar sparing of rectum and bladder. A worse result was found for the bulbus of the penis, due to the helical delivery of HT that is generally associated to a less steep gradient in the cranialcaudal direction compared to conventional delivery systems [16].

A particularly interesting finding of our experience is the high efficiency of our "as low as possible" approach in reducing the integral dose of the OARs surrounding the prostate, like rectum and bladder, or the pelvic nodes, like the bowel. The reduction of the fraction of rectum receiving intermediate-low doses may be one of the causes of the very mild acute GI toxicity profiles experienced by both TOMOADV
[3] and TOMOSIB [17] patients, as suggested by recent investigations about dose-volume relationships for acute rectal toxicity (see for instance [18]), suggesting a parallel behaviour for this endpoint.

Our approach in optimizing HT was also particularly efficient in the treatment of pelvic nodes, translating in a dramatic reduction of acute bowel and bladder toxicity compared to conventional box technique [2]. The availability of IMRT combined with planning approaches as the one followed at our Institution may drastically and rapidly change the attitude of a number of physicians who may be reluctant to extend pelvis irradiation in their clinical practice due to the relatively bad impact on quality of life of 4-fields box technique, especially due to upper GI toxicity [19].

The application of daily MVCT based correction of set-up deserves some additional consideration. The recent advent of Image-guided Radiotherapy (IGRT) for the treatment of prostate cancer is mainly due to the proximity of the prostate to organs at risk, primarily the rectum, in a "moving" organ scenario.

A distended rectum at the planning scan was found to significantly reduce local control [20], due to a systematic difference between planned and actual position of the prostate during treatment, with a consequent missing of the target, suggesting the need for IGRT in prostate radiotherapy. On the other hand, procedures that limit the variation of rectal filling may potentially reduce prostate motion "a priori". The analysis of a quite large sample of our TOMOSIB patients demonstrated the high efficiency of a quite easy and cheap method, as rectal enema, in dramatically reducing the impact of prostate motion and deformation. This result is in agreement with a number of recent papers reporting significantly reduced motion of rectum/prostate with using diet and/or laxatives and/or rectal enema/rectal wash [12,21-23].

In our opinion, these findings suggest that the impact of organ motion may be drastically reduced even in those centers where IGRT technology is not available. Moreover, the application of strategies to reduce the impact of variable rectal filling should be considered a good practice also in the centers using IGRT: a large rectum can deform the prostate (and still more the seminal vesicles shape) and consequently reduce the efficiency in correcting prostate motion through IGRT with a rigid shift approximation. In our opinion, it is much more cost effective to reduce a priori the potential causes of variable rectal filling (and consequently prostate motion) than using IGRT to correct for it a posteriori. 
On the other hand, it is important to underline that the efficiency of rectal emptying procedures (as rectal enema or diet/laxatives) may differ from country to country due to different diet habits and styles of life.

Some additional discussion should be done about the uncertainty in assessing prostate position with MVCT using HT.

The assessment of prostate motion was consistent among different physicians. The two-step procedure with an initial automatic BM before final adjustment by DV may help operators achieve greater consistency as they start from the same "automatically assessed" starting point. This procedure introduces a sort of "bias" as small shifts (i.e.: $<3 \mathrm{~mm}$ ) of the prostate relative to bony anatomy are difficult to assess, as demonstrated by our clinical practice and by the here reported deviations between DV and IPC match. As a consequence, the physician may accept the bony match as representative of the true prostate position in many cases, with, consequently, a slight inter-observer variability. On the other hand, it is evident from our clinical experience that "significant" shifts ( $3 \mathrm{~mm}$ or more) are clearly visible; thus, the reported results must be considered as reliable from the point of view of prostate localization within $3 \mathrm{~mm}$.

An important point concerns the potential reduction of the margins due to the application of IGRT. In our experience, due to the intrinsic uncertainty of the system in detecting small shift and to the existence of other sources of error (as intra-fraction prostate motion [24] and contouring [25]) it seems to be hard to reduce the margins below $5-6 \mathrm{~mm}$. At this moment, we decided to keep the "safe" margins used for 3DCRT ( $8 \mathrm{~mm}$ for LR and PA, $10 \mathrm{~mm}$ for $\mathrm{CC})$; the very mild toxicity GI profile together with the fact that GU toxicity is mainly due to the portion of bladder neck/urethra unavoidably included in the irradiated volumes, suggest that, in our SIB approach, a margin reduction would probably not translate in a reduction of toxicity.

On the other hand, thanks to daily MVCT-based correction, a reduced margins $(5 \mathrm{~mm})$ will be applied in a pilot study of dose escalation to dose $>90$ Gy to dominant intra-prostatic lesions imaged through T2 MRI combined with diffusion imaging, actually under pre-clinical investigation at our Institution; in this case, the reduction of the margin together with a careful application of IGRT is of paramount importance in order to reduce the risk of severe late rectal bleeding.

Declaration of interest. The authors report no conflicts of interest. The authors alone are responsible for the content and writing of the paper.

\section{References}

[1] Mackie TR, Kapatoes J, Ruchala K, Lu W, Wu C, Olivera G, et al. Image guidance for precise conformal radiotherapy. Int J Radiat Oncol Biol Phys 2003;56:89-105.

[2] Cozzarini C, Fiorino C, Di Muzio N, Alongi F, Broggi S, Cattaneo GM, et al. Significant reduction of acute toxicity following pelvic irradiation with Helical Tomotherapy in patients with localized prostate cancer. Radiother Oncol 2007;8:164-70.

[3] Cozzarini C, Fiorino C, Di Muzio N, Valdagni R, Salonia A, Alongi $\mathrm{F}$, et al. Hypofractionated adjuvant radiotherapy with helical tomotherapy after radical prostatectomy: Planning data and toxicity results of a phase I-II study. Radiother Oncol 2008;88:26-33.

[4] Bentzen SM, Baumann M. The linear quadratic model in clinical practice. In: Basic Clinical Radiobiology. Steel GG, editor. London (UK): Arnold Editions; 2002. p 134-146.

[5] Fiorino C, Sanguineti G, Cozzarini C, Fellin G, Foppiano F, Menegotti L, et al. Rectal dose-volume constraints in highdose radiotherapy of localized prostate cancer. Int J Radiat Oncol Biol Phys 2003;57:953-62.

[6] Fiorino C, Fellin G, Rancati T, Valvassori V, Bianchi C, Casanova Borca V, et al. Clinical and dosimetric predictors of late rectal syndrome after 3DCRT for localized prostate cancer: Preliminary results of a multicenter prospective study. Int J Radiat Oncol Biol Phys 2008;70:1130-7.

[7] Nahum AE, Movsas B, Horwitz EM, et al. Incorporating clinical measurements of hypoxia into tumor local control modeling of prostate cancer: implications for the alpha/beta ratio. Int J Radiat Oncol Biol Phys 2003;572:391-401.

[8] Shepard DM, Olivera GH, Reckwerdt PJ, Mackie TR. Iterative approaches to dose optimization in tomotherapy. Phys Med Biol 2000;45:69-90.

[9] Di Muzio N, Fiorino C, Cozzarini C, Alongi F, Broggi S, Mangili $\mathrm{P}$, et al. Hypofractionated simultaneous integrated boost tomotherapy in localized prostate cancer: Preliminary toxicity results. Int J Radiat Oncol Biol Phys 2007;69(Suppl):S367-S368 (Abstract).

[10] Sanguineti G, Little M, Endres EJ, et al. Comparison of three strategies to delineate the bowel for whole pelvis IMRT of prostate cancer. Radiother Oncol 2008;88:95-101.

[11] Broggi S, Fiorino C, Maggiulli, Di Muzio N, Cozzarini C, Cattaneo GM, et al. On-line and off-line correction strategies using MVCT in prostate cancer Helical Tomotherapy. Proceeding of $\mathrm{XV}^{\circ}$ ICCR Congress 2007; 400-4.

[12] Fiorino C, Di Muzio N, Broggi S, Cozzarini C, Maggiulli E, Alongi F, et al. Evidence of limited motion of the prostate by carefully emptying the rectum as assessed by daily MVCT image guidance with helical tomotherapy. Int J Radiat Oncol Biol Phys 2008;71:611-7.

[13] Iori M, Cattaneo GM, Cagni E, Fiorino C, Borasi G, Calandrino $\mathrm{R}$, et al. Dose-volume and biological model based comparison between helical tomotherapy and (inverse-planned) IMAT for prostate tumours. Radiother Oncol 2008;88:34-45.

[14] Grigorov G, Kron T, Wong E, Chen J, Sollazzo J, Rodrigues G. Optimization of helical tomotherapy treatment plans for prostate cancer. Phys Med Biol 2003;48: 1933-43.

[15] Rodrigues G, Yartsev S, Chen J, Wong E, Souza DD, Lock $\mathrm{M}$, et al. A comparison of prostate IMRT and helical tomotherapy class solutions. Radiother Oncol 2006;80: 374-7.

[16] Perna L, Mangili P, Fiorino C, Di Muzio N, Cozzarini C, Broggi S, et al. Sparing the penile bulbs by MRI-based 
prostatic apex definition in 3DRCT and Helical Tomotherapy of prostate cancer. Radiother Oncol 2006;81(Suppl 1):S45 (Abstract).

[17] Di Muzio N, Fiorino C, Cozzarini C, Alongi F, Broggi S, Mangili P, et al. Phase I-II study of hypofractionated simultaneous integrated boost with tomotherapy for prostate cancer. Int J Radiat Oncol Biol Phys (Submitted)

[18] Vavassori V, Fiorino C, Rancati T, Magli A, Fellin G, Baccolini $M$, et al. Predictors for rectal and intestinal acute toxicities during prostate cancer high-dose 3D-CRT: Results of a prospective multicenter study. Int J Radiat Oncol Biol Phys 2007;66:1401-10.

[19] Dirix P, Haustermans K, Junius S, Withers R, Oyen R, Van Poppel H. The role of whole pelvic radiotherapy in locally advanced prostate cancer. Radiother Oncol 2006;79: $1-14$.

[20] De Crevoisier R, Tucker SL, Dong L, Mohan R, Cheung R, Cox JD, et al. Increased risk of biochemical and local failure in patients with distended rectum on the planning CT for prostate cancer radiotherapy. Int J Radiat Oncol Biol Phys 2005;62:965-73.
[21] Stasi M, Munoz F, Fiorino C, Pasquino M, Baiotto B, Marini $P$, et al. Emptying the rectum before treatment delivery limits the variations of rectal dose-volume parameters during 3DCRT of prostate cancer. Radiother Oncol 2006;80:363-70.

[22] Nijkamp J, Pos FJ, Nuver TT, De Jong R, Remeijer P, Sonke $\mathrm{JJ}$, et al. Adaptive radiotherapy for prostate cancer using kilovoltage con-beam computed tomography: First clinical results. Int J Radiat Oncol Biol Phys 2008;70:75-82.

[23] Ogino I, Uemura $H$, Inoue $T$, Kubota $Y$, Nomura $K$, Okamoto N. Reduction of prostate motion by removal of gas in rectum during radiotherapy. Int J Radiat Oncol Biol Phys 2008 (in press).

[24] Litzenberg DW, Balter JM, Hadley SW, Sandler HM, Willoughby TR, Kupelian PA, et al. The influence of intrafraction motion on margins for prostate radiotherapy. Int J Radiat Oncol Biol Phys 2006;65:548-53.

[25] Fiorino C, Reni M, Bolognesi A, Cattaneo GM, Calandrino $\mathrm{R}$. Intra and inter-observer variability in contouring prostate and seminal vesicles: Implications for conformal treatment planning. Radiother Oncol 1998;47:285-92. 